\title{
Las brechas digitales en Dabeiba y Frontino, Colombia: un desafío para la educación virtual para la paz
}

María Constanza Torres-Madroñero'

Instituto Tecnológico Metropolitano -ITM

mariatorres@itm.edu.co

Esperanza Milena Torres-Madroñero²

Institución Universitaria Colegio Mayor de Antioquia

esperanza.torres@colmayor.edu.co

DOI: https://doi.org/10.21158/2357514x.v8.n2.2020.2719

Cómo citar este artículo: Torres-Madroñero, M. C.; Torres-Madroñero, E. M. (2020). Las brechas digitales en

Dabeiba y Frontino, Colombia: un desafío para la educación virtual para la paz. Revista Virtu@lmente, 8(2), 101-114. DOI: https://doi.org/10.21158/2357514x.v8.n2.2020.2719

Fecha de recepción: 08 de agosto 2020

Fecha de aprobación: 21 de septiembre 2020

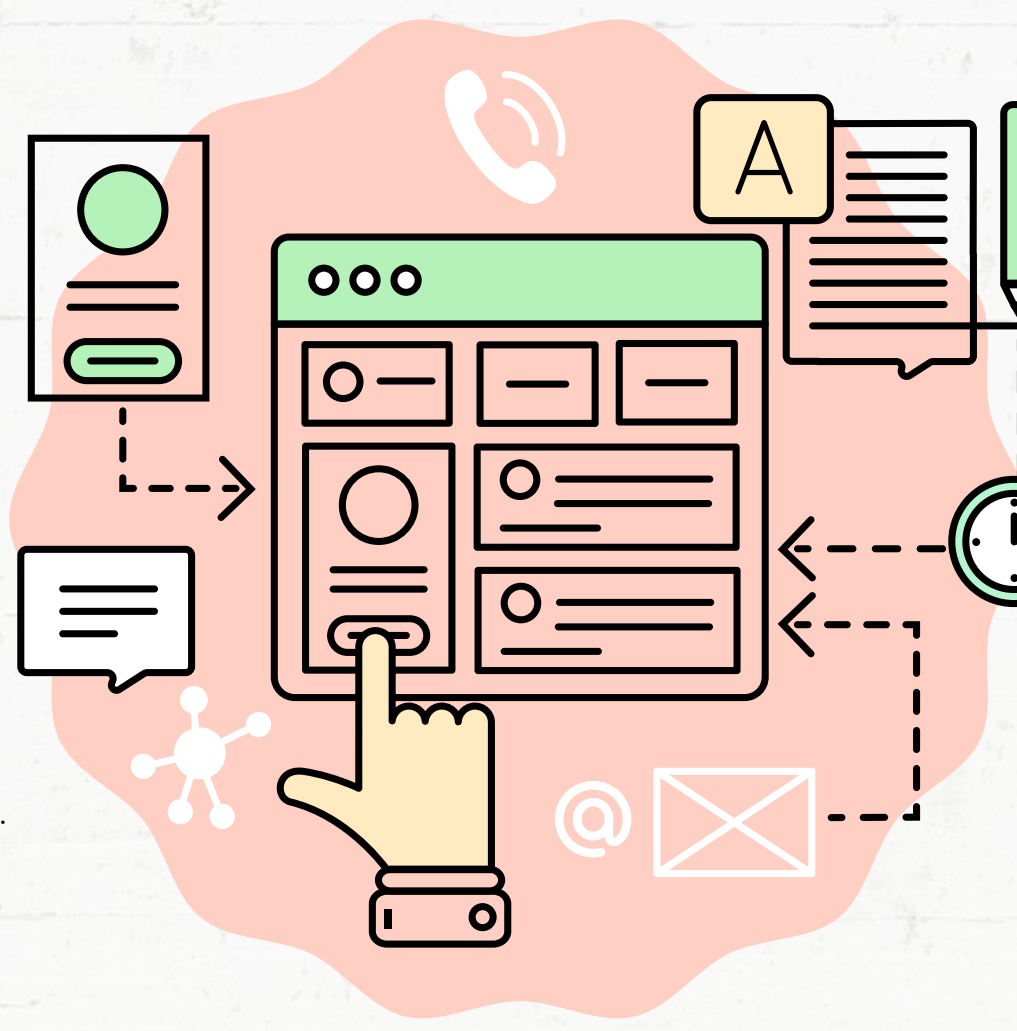

$\overline{1}$ Doctora en Ciencias e Ingeniería de la Información y la Computación de la Universidad de Puerto Rico, con Maestría en Ingeniería eléctrica de la misma universidad. Pregrado en Ingeniería Electrónica de la Universidad Nacional de Colombia, sede Manizales. ORCID: https://orcid.org/0000-0002-9795-2459

${ }^{2}$ Candidata a doctora en Ciencias Sociales y Humanas de la Universidad Nacional de Colombia, sede Medellín. Maestría en Comunicación con énfasis en teorías e investigación de la Universidad de Puerto Rico. Pregrado en Sociología de la Universidad de Nariño. ORCID: https://orcid.org/0000-0002-4711-756X 


\section{RESUMEN}

Este es un estudio de casos centrado en la incorporación y apropiación de las Tecnologías de la Información y la Comunicación (TIC) para la educación en la ruralidad colombiana. Desde la implementación de proyecto PAZRED y su objetivo de construir una cultura de paz desde una apuesta virtual y pedagógica, se han identificado diferentes dimensiones de las brechas digitales en los municipios de Dabeiba y Frontino, en el Occidente antioqueño. Estas dimensiones se encuentran relacionadas con el acceso y uso de las tecnologías, el acompañamiento en los procesos de aprendizaje y la incorporación de la multiculturalidad de la población indígena. Este artículo expone el estado actual de las brechas digitales en estas dos zonas, así como los desafíos que presenta la educación virtual en estos escenarios. La metodología implementada en el proceso fue de carácter cualitativo, principalmente a través de entrevistas semiestructuradas, observación sistemática y revisión documental. De esta manera, se pudo dar cuenta de las brechas digitales en las zonas de estudio en términos de acceso, calidad y complejidad que se demanda para la educación en contextos con amplia diversidad cultural y de carácter rural. El análisis concluye que es necesario ampliar el concepto de "cobertura tecnológica», puesto que la apropiación tecnológica por parte de las comunidades rurales e indígenas debe ir más allá de la incorporación de TIC. Se requiere generar contenidos propios, modelos educativos y comunicativos con soporte tecnológico posicionados ética y políticamente, que reflejen las necesidades de aprendizaje, comprendan las lógicas y lenguajes propios de las comunidades.

Palabras clave: educación rural; educación virtual; brechas digitales; brechas tecnológicas; cobertura tecnológica; apropiación tecnológica; multiculturalidad en la educación; cultura de paz; educación para la paz. 


\section{The digital gaps in Dabeiba and Frontino, Colombia: a challenge for virtual peace education}

\section{ABSTRACT}

This is a case study focusing on the incorporation and appropriation of Information and Communication Technologies (ICTs) for education in rural Colombia. Ever since implementing the PAZRED project and its objective of building a culture of peace from a virtual and pedagogical approach, different dimensions of the digital gap have been identified in the municipalities of Dabeiba and Frontino, in Western Antioquia. These dimensions are related to access and use of technologies, the accompaniment in the learning processes, and the incorporation of the multiculturalism of the indigenous population. This article presents the current state of the digital gap in these two areas and the challenges of virtual education in these scenarios. We implemented in the process a qualitative methodology, mainly through semi-structured interviews, systematic observation, and documentary review. In this way, it was possible to identify the digital gaps in the areas of study in terms of access, quality, and complexity required for education in contexts with wide cultural diversity and of rural character. The analysis concludes that it is necessary to broaden the concept of "technological coverage", since technological appropriation by rural and indigenous communities must go beyond the incorporation of ICTs. It is necessary to generate suitable content educational and communication models with technological support, positioned ethically and politically, that not only reflect their learning necessities but also understand the logic and the language of these communities.

Keywords: rural education; virtual education; digital gaps; technological gaps; technological coverage; technological appropriation; multiculturalism in education; culture of peace; education for peace. 


\section{As aberturas digitais em Dabeiba e Frontino, Colômbia: um desafio para a educação virtual para a paz}

\section{RESUMO}

Este é um estudo de casos focado na incorporação e apropriação das Tecnologias da Informação e Comunicação (TIC) para a educação na zona rural colombiana. Desde a implantação do projeto PAZRED e seu objetivo de construir uma cultura de paz a partir de uma aposta virtual e pedagógica, foram identificadas diferentes dimensões das aberturas digitais nos municípios de Dabeiba e Frontino, no oeste da Antioquia. Essas dimensões estão relacionadas ao acesso e uso de tecnologias, ao acompanhamento nos processos de aprendizagem e à incorporação da multiculturalidade da população indígena. Este artigo apresenta o estado atual das aberturas digitais nessas duas áreas, bem como os desafios que a educação virtual apresenta nesses lugares. A metodologia implementada no processo foi de natureza qualitativa, principalmente através de entrevistas semiestruturadas, observação sistemática e revisão documental. Desta forma, foi possível perceber as aberturas digitais nas áreas de estudo em termos de acesso, qualidade e complexidade que se exige para a educação em contextos de grande diversidade cultural e rural. A análise conclui que é necessário ampliar o conceito de "cobertura tecnológica", visto que a apropriação tecnológica pelas comunidades rurais e indígenas deve ir além da incorporação das TIC. É necessário gerar conteúdos próprios, modelos educacionais e comunicativos com suporte tecnológico que se posicionem ética e politicamente, que reflitam as necessidades de aprendizagem e entendam as lógicas e as linguagens próprias das comunidades.

Palavras-chave: educação rural; educação virtual; aberturas digitais; aberturas tecnológicas; cobertura tecnológica; apropriação tecnológica; multiculturalismo na educação; cultura de paz; educação para a paz. 


\section{Fractures numériques à Dabeiba et Frontino: défi pour l'éducation virtuelle pour la paix en Colombie}

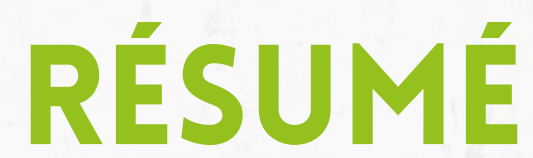

Cette étude de cas est axée sur l'incorporation et l'appropriation des technologies de l'information et de la communication pour l'éducation (TICE) dans la Colombie rurale. Dès la mise en œuvre du projet PAZRED et de l'objectif de construction de la paix à partir d'un engagement pédagogique virtuel, différentes fractures numériques ont été identifiées dans les communes de Dabeiba et Frontino, dans l'ouest du département d'Antioquia. Ces fractures sont liées à l'accès et à l'utilisation des technologies numériques, à l'accompagnement des processus d'apprentissage et à l'intégration du multiculturalisme de la population autochtone. Cet article présente l'état actuel de la fracture numérique dans ces deux régions et les défis posés par l'éducation virtuelle. La méthodologie utilisée est de type qualitative, réalisée au travers d'entretiens semi-structurés, d'observation systématique et documentée. II a ainsi été possible de rendre compte des fractures numériques des régions étudiées en termes d'accès, de qualité et de complexité éducative dans des contextes de grande diversité culturelle et de ruralité profonde. L'analyse conclut qu'il est indispensable d'élargir le concept de «couverture technologique» puisque I'appropriation des technologies par les communautés rurales et autochtones doit aller au-delà de l'incorporation des TICE. II est ainsi nécessaire de créer des contenus propres, des modèles éducatifs et communicatifs axés sur la technologie, prenant compte des défis éthiques et politiques, reflétant les besoins d'apprentissage, et comprennant la logique et les langues des communautés.

Mots-clés: éducation rurale; éducation virtuelle; fractures numériques; fractures technologiques; couverture technologique; appropriation technologique; multiculturalisme dans l'éducation; culture de la paix; éducation pour la paix. 


\section{Introducción}

El proyecto de investigación «Cátedra virtual de paz - PAZRED: tejidos colectivos derivados de la memoria en el Occidente de Antioquia» es una apuesta por integrar la investigación en procesos de enseñanza y aprendizaje en la cultura de paz, a través de una plataforma digital creada desde la reconstrucción de la memoria colectiva de los municipios de Dabeiba y Frontino. A partir de la implementación de este proyecto, se identificaron problemáticas, desafíos y realidades alrededor de la implementación de la educación virtual en zonas rurales del país. La incorporación de las Tecnologías de la Información y la Comunicación (TIC) en los procesos de enseñanza y aprendizaje se promueven como un mecanismo para incrementar los indicadores de accesibilidad a la educación en todos los niveles de formación (Unesco, 1998). Sin embargo, este proceso tiene diversas limitaciones, entre las que se encuentran las brechas digitales, las cuales se acentúan más en contextos rurales.

El término «brecha digital» tiene una amplia connotación en la literatura. Este se refiere no solo a la limitación de acceso a las TIC, ya sea por situaciones económicas, sociales o geográficas, sino también a la limitación o al no uso estas tecnologías, aunque se cuente con acceso a ellas, debido a la falta de conocimiento sobre su utilización o poca apropiación de estas. Así mismo, «brecha digital» se asocia a la calidad de las actividades que se logra desarrollar con las TIC. La primera dimensión se soluciona con desarrollo de infraestructura y programas de cobertura digital; la segunda y tercera, por su parte, se solucionan a partir de programas educativos y capacitación (Cabero-Almenara y Ruiz-Palmero, 2017).
La brecha digital se acentúa mucho más en poblaciones originarias y afrodescendientes, como lo plantea la Comisión Económica para América Latina y el Caribe (Cepal), que afirma que estas poblaciones tienen una probabilidad cinco veces menor de acceder a computadores y conectividad, relacionado con los niveles educativos; incluso en las zonas urbanas, estos grupos poblacionales tienen una menor probabilidad de acceso (Villatoro y Silva, 2005). En el caso de las comunidades indígenas, se suma la exclusión de otras formas de saber, prácticas de lectura y escritura diferentes a las occidentales. Las TIC inciden en la reproducción de la desigualdad, no solo en términos de la apropiación tecnológica y la conectividad que requieren, sino por el desconocimiento y la invisibilización de otras formas de lectura y escritura que conllevan, que omiten, en muchos casos, esquemas de comunicación orales presentes en las comunidades indígenas, rurales y afrodescendientes.

Este articulo tiene el objetivo de analizar las brechas digitales en dos municipios del Occidente antioqueño en Colombia: Dabeiba y Frontino. Ambos municipios rurales cuentan con población indígena y han sido víctimas del conflicto armado del país; por tanto, se analiza como caso de estudio, para establecer las limitaciones y oportunidades de la educación virtual en territorios rurales del país. Con este trabajo se busca responder la pregunta de investigación: ¿cuáles son los factores limitantes en la incorporación de las TIC en los procesos educativos de poblaciones rurales en Colombia? 
La metodología implementada en el proceso fue de carácter cualitativo, principalmente a través de entrevistas semiestructuradas, observación sistemática y revisión documental. De esta manera, se pudo dar cuenta de las brechas digitales en las zonas de estudio en términos de acceso, calidad y complejidad que se demanda para la educación en contextos con amplia diversidad cultural y de carácter rural.

El documento que se presenta da cuenta del proceso metodológico, que incluye una descripción de las zonas de estudio, la exposición de los instrumentos de construcción de información y la contextualización de la información poblacional, y de otro tipo, que ayuda a ubicar el problema de investigación. En seguida, se presentan los principales resultados, que contemplan una discusión en términos de lo que se entiende por conectividad, la integración tecnológica de las comunidades indígenas como una necesidad a partir de la Constitución de 1991 en Colombia y los focos de acción que han tenido las diferentes políticas públicas en términos de tecnologías de la información en relación con lo educativo. En la sección de discusión se presentan los desafíos al contemplar un concepto amplio de «conectividad» que incluya una lectura desde las particularidades culturales y sociales.

\section{Metodología}

Para establecer las brechas digitales existentes en los municipios de Dabeiba y Frontino, se estudiaron las cifras de analfabetismo, acceso a conexión a internet y propuestas de educación virtual en la región, comparadas con el número de habitantes de la zona y su caracterización. Como parte del proyecto de investigación PAZRED, se realizaron visitas a las zonas de estudios, donde se pudo constatar el acceso a las TIC.

\subsection{Instrumentos para la construcción de información}

La investigación fue de carácter cualitativo, se centró en la significatividad de la información a partir de la particularidad de los datos; no tuvo pretensiones de generalidad, sino de concentrar esfuerzos en los actores y en el contexto particular de las zonas de estudio. Para alcanzar los resultados, se implementaron entrevistas semiestructuradas y se seleccionaron los actores a partir de un muestreo no probabilístico; de igual manera, se implementaron la observación y un diario de campo para reconocer algunas características de la conectividad en la zona. De forma simultánea, se realizó un proceso de documentación.

\subsection{Zonas de estudio}

Para este estudio se analizó la situación de acceso tecnológico de los municipios de Dabeiba y Frontino (Figura 1), pertenecientes a la subregión del Occidente antioqueño en Colombia. Esta subregión se localiza entre las cordilleras Central y Occidental, y está conformada por 19 municipios ubicados en la cuenca media del río Cauca y en las cuencas de los ríos Sucio y Murrí. 
Figura 1. Geolocalización de los municipios de Dabeiba y Frontino en el Occidente de Antioquia, Colombia

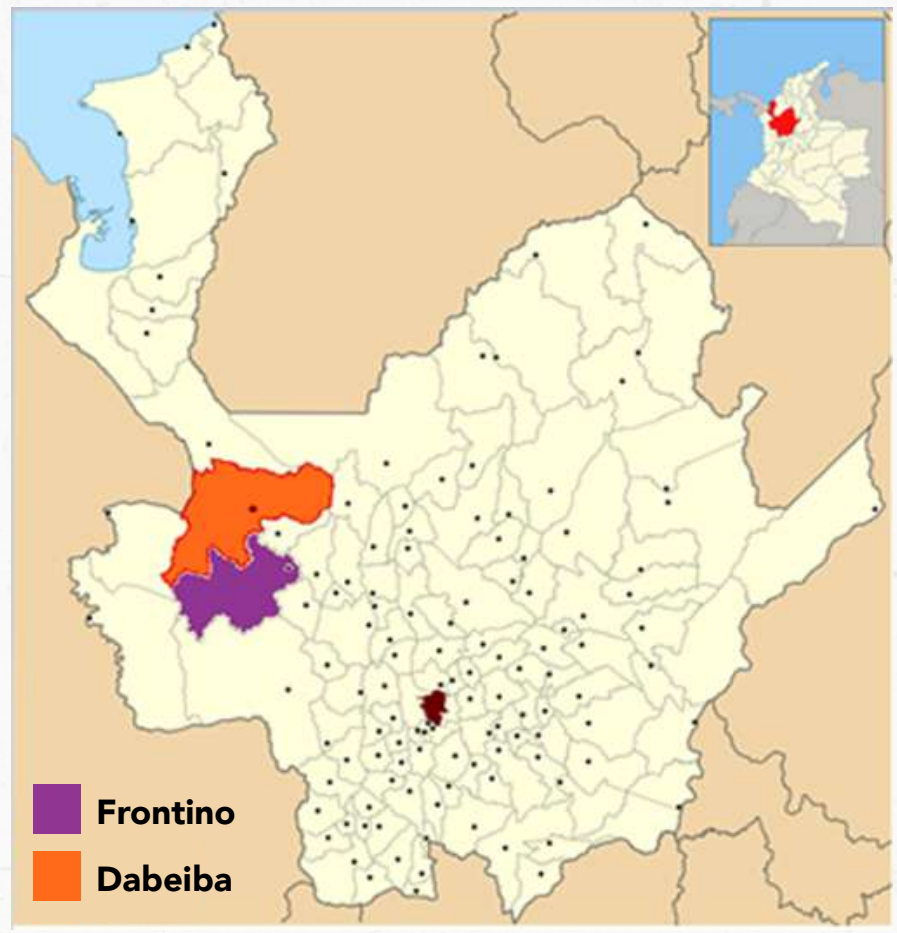

Fuente. Adaptación de imagen de Wikimedia Commons

Frontino es reconocido como municipio desde 1850. Su economía se basa en la agricultura, la ganadería y la minería. Los principales cultivos de la zona son la caña de azúcar y el café, y en menor medida, el maíz y el frijol. Sus pobladores también se dedican a la ganadería de carne y la producción lechera. Sin embargo, la actividad económica más importante es la extracción de oro y cobre. De acuerdo con la proyección del Departamento Administrativo Nacional de Estadística
(DANE, 2020), el número de habitantes del municipio de Frontino para 2020 es de 20.700, con 9.020 en la cabecera municipal y 11.680 en centro poblado y rural disperso. De acuerdo con el Censo Nacional de Población y Vivienda de 2018, el 10,19 \% de la población de Frontino reside en vivienda tradicional indígena (DANE, 2018). 
Por su parte, el municipio de Dabeiba, fundado el 3 de noviembre de 1850, tiene como actividades económicas la agricultura, la ganadería, la minería, la exploración forestal y el comercio. En la producción agrícola se destacan el café, el cacao, el frijol, el aguacate, el maíz, el lulo y el maracuyá, entre otros; en la ganadería, la carne de res y los productos lácteos. De acuerdo con la proyección del DANE (2020), Dabeiba cuenta con 23.509 habitantes para 2020, con 9.380 en la cabecera municipal y 14.129 en la zona rural. De acuerdo con el censo de 2018 , el 8,82 \% reside en vivienda tradicional indígena (DANE, 2018).

Tanto Dabeiba como Frontino se caracterizan por ubicarse en un corredor estratégico que comunica el interior de Antioquia con la salida al mar, de modo que demarcan una ruta importante para el transporte de mercancía. A su vez, este ha sido el detonante de una prolongada lucha territorial entre grupos armados legales e ilegales, que se disputan el control de estas zonas. A esta condición se le suma la riqueza natural y mineral de estos territorios, que los ha convertido en escenarios de interés para megaproyectos como la construcción del túnel del Toyo, que hace parte de las Autopistas para la Prosperidad lideradas por el Gobierno nacional.

\subsection{Alfabetización y cobertura educativa}

De acuerdo con el censo de 2018, el 14,61 \% de la población de Frontino no sabe leer y escribir; para el caso de Dabeiba, esta cifra es del 19,43\% (DANE, 2018).
La población de Frontino cuenta con diez instituciones educativas para primaria, secundaria y educación media, de acuerdo con el MEN (2020). De estas instituciones, seis están ubicadas en la cabecera municipal, dos en veredas, una en corregimiento y una en un resguardo. Por su parte, el municipio de Dabeiba cuenta con seis instituciones educativas activas: dos en la cabecera municipal, dos en veredas y dos en corregimientos.

En cuanto a educación superior, ninguno de los municipios de las zonas de estudio cuenta con instituciones registradas en el Sistema Nacional de Información de Educación Superior (SNIES) del MEN. Sin embargo, en la región se han desarrollado iniciativas para articular la población con instituciones de educación superior. Por ejemplo, finalizando la década de 1990 e iniciando la del 2000, se desarrollaron programas a distancia en Frontino con el Politécnico Marco Fidel Suárez, la Corporación Universitaria Remington y la Universidad de Pamplona. Además, en 2017 la universidad a distancia Areandina graduó a estudiantes de comunidades indígenas de Frontino de la Licenciatura en Pedagogía Infantil. Actualmente, el Servicio Nacional de Aprendizaje (Sena) tiene presencia en el territorio con escuelas en agropecuaria y salud. De acuerdo con la Secretaría de Educación de Dabeiba, solo EDUPOL ${ }^{3}$ tiene presencia en este municipio, una empresa de origen brasileño que oferta programas académicos virtuales.

${ }_{3}^{3}$ Para ampliar información, véase https://edupol.com.co/quienes-somos/ 


\subsection{Conectividad}

De acuerdo con el censo de 2018, el 54,7 \% de los hogares en Antioquia tiene conexión a internet. En las cabeceras municipales, la cifra de hogares con internet es del 66,2\%, pero en las zonas rurales del departamento esta es apenas del $12,4 \%$. Por otra parte, el $42,0 \%$ afirma que no sabe usar internet (DANE, 2018).

\section{Resultados}

La conectividad involucra dimensiones que trascienden la tenencia de computadores y de internet. La mirada que ha prevalecido limita la comprensión de la tecnología como un instrumento y no como un componente que emerge y altera las dimensiones sociales de las que es parte. La conectividad, desde una mirada compleja, tiene que ver con el acceso físico a la tecnología, la apropiación, la alfabetización y la comprensión de los contextos sociales, culturales y económicos donde tiene lugar.

El acceso físico a la tecnología es la posibilidad de tener instrumentos que faciliten el intercambio de información y la comunicación con otras y otros. La aplicación de la tecnología apropiada para las condiciones locales en términos culturales, políticos, económicos y sociales; la generación de capacidades; el desarrollo de contenido local relevante; la integración de la tecnología a las rutinas diarias, y la confianza en las TIC, entre otros (Betancourt, 2004).
Según el Censo Nacional de Población y Vivienda (CNPV) 2018, la población que se autoreconoce como indígena en el país es de 1.905.617. En el occidente de Antioquia, Dabeiba y Frontino, se concentra gran cantidad de esta población, especialmente embera, que se encuentra excluida de los desarrollos tecnológicos, no solo por la cobertura, sino por limitaciones lingüísticas y culturales.

En relación con esta población, a partir de la Constitución de 1991 se reconoce la ciudadanía a todas las comunidades indígenas. Esto ha traído consigo la incorporación de esfuerzos institucionales por integrar al uso tecnológico a las 102 comunidades indígenas que tienen presencia en el país. Esta demanda condujo a políticas de inclusión tecnológica que se debatían en la paradoja entre la conectividad física y el desconocimiento de las particularidades de las comunidades indígenas.

Como señalan Cadavid-Bringe, Herrera-Huérfano, FayadSanz y Vega-Casanova (2017), la discriminación de los pueblos indígenas después de la colonización se basó en la idea de que el carácter de ciudadanía de las comunidades indígenas se resolvería mediante su incorporación al proyecto de la civilización. Como enuncian estos mismos autores se trataba de discursos y políticas de «blanqueamiento cultural» mediante el ordenamiento jurídico (Cadavid-Bringe et al., 2017 , p. 151). En este sentido, las TIC operaban como uno de los elementos del proyecto moderno y civilizatorio que se reflejaba especialmente en las apuestas de educación formal. 
El actual marco normativo colombiano reconoce en la Constitución de 1991 que las comunidades indígenas tienen derecho al mantenimiento de sus riquezas culturales, a la preservación de su lengua y dialectos, a la propiedad comunitaria, a la ley propia, a la autogestión, a la participación política y a una enseñanza que mantenga las tradiciones y saberes propios. Sin embargo, en el contexto de la tecnologización educativa, estos aspectos continúan rezagados, el acceso a las TIC está concentrado en las zonas urbanas con ciertos niveles de desarrollo y la apropiación de la tecnología no permite aún la generación de discursos propios educativos que respondan a la diversidad étnica del país y la región.

A pesar de ello, diversos esfuerzos institucionales han intentado dar respuesta a la exigencia constitucional. El primero se enmarcaba en la radiodifusión a través del Programa Comunidad en 1999 del Ministerio de Comunicación, hoy denominado Ministerio de las Tecnologías de la Información y las Comunicaciones (MinTIC). Entre 1998 y 2009 se implementó una política pública en telecomunicaciones sociales - Compartel- que, junto con los planes nacionales de desarrollo, buscaba ampliar el acceso.

Entre 2002 y 2006 se asignaron 25 emisoras de carácter comunitario a los pueblos indígenas. Se implementaron 2000 telecentros en el plan Compartel y telecentros comunitarios. Esto como evidencia de la permanente búsqueda institucional por ampliar la cobertura tecnológica, en especial en los territorios rurales e indígenas. Actualmente se distinguen proyectos como Puntos Vive Digital, conectividad para las instituciones públicas, telecentros, telefonía rural y Nuevo Compartel (MinTIC, 2012, citado en Cadavid-Bringe et al., 2017, p. 152).

El Plan TIC 2018-2022 del MinTIC (2018a) tiene entre sus metas la inclusión social digital y la apropiación de las TIC por parte de la ciudadanía. Entre las estrategias para la inclusión social digital se encuentran: el aumento de espacios públicos de conexión gratuita a internet, denominados «zonas digitales urbanas»; un programa para masificar el acceso universal orientado principalmente a los centros de zonas rurales; el desarrollo de un instrumento para medir la brecha digital en el país, y el despliegue de infraestructura para incrementar la calidad y cobertura de los servicios de telecomunicaciones. Por otra parte, entre las estrategias para la apropiación de las TIC se encuentran: la iniciativa "ciudadanía digital», para la capacitación en competencias para el uso de las TIC en todos los niveles. A partir del Plan TIC 2018-2022 se deriva el Plan Nacional de Conectividad Rural 2018-2022 (MinTIC, 2018b), el cual se centra en el despliegue de infraestructura con el fin de garantizar el acceso a internet en cabeceras municipales - priorizando centros poblados de más de cien habitantes-.

Por su parte, el Ministerio de Educación Nacional de Colombia (MEN) en su plan estratégico 2019-2022 (MEN, 2019) define como estrategias para impulsar la educación superior incluyente y de calidad las siguientes: potenciar alternativas de educación superior, que respondan a las necesidades y características de lo rural, y ampliar la oferta de programas virtuales y a distancia que contribuyan a la cobertura, acceso y permanencia de jóvenes. 


\section{Discusión}

A partir de las realidades de los municipios de Dabeiba y Frontino, se pueden identificar cuatro factores que limitan el aprovechamiento de las TIC para procesos formativos en zonas rurales del país: (a) el acceso a la tecnología, (b) los conocimientos y competencias para su uso y aprovechamiento, (c) la falta de reconocimiento de la multiculturalidad y diversidad lingüística, y (d) la coherencia de las tecnologías con las actividades económicas de la región.

A pesar de los esfuerzos nacionales realizados, aún existe una amplia brecha que superar en relación con el acceso a las TIC. Son pocos los hogares en las regiones rurales con acceso a internet y computador - $\mathrm{u}$ otros dispositivos móviles-. En Colombia esta brecha se está tratando de solucionar desde el Plan Nacional de Conectividad Rural 2018-2022. Sin embargo, este se limita a las cabeceras municipales $y$, por tanto, se excluye a los territorios rurales dispersos.

Por otra parte, reconociendo que las poblaciones de estas zonas no alcanzan la educación superior, no solo es necesario solucionar el acceso a infraestructura, sino también la apropiación del uso de las TIC mediante el desarrollo de competencias que le permitan a la comunidad su aprovechamiento. Sobre este punto, no son claros los programas y planes nacionales existentes.

Un tema que poco se ha tenido en cuenta en el desarrollo de planes para la incorporación de las TIC es la multiculturalidad y diversidad de lenguajes presentes en las zonas rurales. El uso de las TIC debe adaptarse a estas poblaciones y no al contrario. Si se busca que las TIC sean una herramienta para ampliar la cobertura y mejorar la calidad educativa en las zonas rurales, como lo promueven las Naciones Unidas (Unesco, 1998) y el MEN (2019), es crucial desarrollar herramientas que adopten los lenguajes propios de cada región y se adapten a sus necesidades. Además, se debe procurar que las herramientas TIC que se adopten en regiones rurales respondan a sus actividades económicas y culturales.

\section{Conclusiones}

Las brechas digitales son parte de las desigualdades sociales vigentes en el modelo económico moderno, esto fragmenta la comprensión de las dinámicas rurales y urbanas, que se entienden como desconectadas entre sí, omitiendo su interdependencia. El modelo de desarrollo ha centrado sus servicios e intervenciones en escenarios urbanos y cabeceras municipales, lo que impide un alcance mayor, especialmente en zonas rurales dispersas.

Las políticas instituidas tienen una lectura que prioriza los indicadores de cobertura tecnológica en términos físicos, pero quedan relegados a un segundo renglón el desarrollo de capacidades, la apropiación y el reconocimiento cultural que involucra la tecnología. Se requiere aunar esfuerzos para proyectar lecturas interdisciplinares, que posibiliten entender la particularidad de los territorios y sus necesidades, 
sobrepasar la intervención pública basada exclusivamente en los indicadores y apostar por lecturas más complejas desde las ciencias sociales.

En consecuencia, es necesario ampliar el concepto de «cobertura tecnológica»: la apropiación tecnológica por parte de las comunidades rurales e indígenas debe ir más allá de la incorporación de TIC. Se requiere generar contenidos propios, modelos educativos y comunicativos con soporte tecnológico posicionados ética y políticamente, que reflejen las necesidades de aprendizaje, comprendan las lógicas y lenguajes propios de las comunidades y contribuyan a forjar horizontes de sentido que rebosen el marco de comprensión del mundo moderno.

En este sentido, de las tecnologías deberán emerger las iniciativas de resistencia social, los lenguajes no convencionales, las apuestas estéticas, las proyecciones de política no formal, modelos educativos flexibles, críticos y emancipadores.

El desafío educativo desde TIC en el occidente de Antioquia demanda entender las relaciones y los discursos de poder de los que son parte las tecnologías. Los valores de la eficiencia, rapidez e interactividad son compartidos con el deber ser económico vigente, de manera que el discurso del acceso tecnológico responde a las dinámicas económicas globalizadas. Esto implica entender las TIC como un escenario simbólico de disputa, donde se debe abrir camino a otras formas de conocer, intercambiar y habitar el mundo. El reconocimiento de la diversidad humana demanda a su vez diversidad de usos tecnológicos en lo educativo. Se trata de entender maneras de comunicar y comprender el mundo que no necesariamente están inmersas en el modelo de desarrollo ni en las lógicas de racionalidad modernas.

Por último, se recomienda desarrollar propuestas tecnológicas que permitan reducir las brechas digitales en sus diferentes dimensiones; no solo encaminadas a incrementar la infraestructura, sino también a fomentar la apropiación de las TIC, incorporando la multiculturalidad de las poblaciones rurales.

\section{Referencias}

Betancourt, V. (2004). El problema de la brecha digital: más allá de las fronteras de la conectividad. Pez de plata: Bibliotecas Públicas a la vanguardia, 1(3).

Cabero-Almenara, J.; Ruiz-Palmero, J. (2017). Las Tecnologías de la Información y Comunicación para la inclusión: reformulando la brecha digital. International Journal of Educational Research and Innovation,(9), 16-30.

Cadavid-Bringe, A; Herrera-Huérfano, E.; Fayad-Sanz, D.; Vega-Casanova, J. (2017). Apropiación de TIC, pueblos indígenas de resistencia en Colombia: el caso del Tejido de Comunicación. En F. Sierra Caballero; T. Gravante (Coord.), Tecnopolítica en América Latina y el Caribe. (145-169). Salamanca: Comunicación Social Ediciones y Publicaciones.

DANE. (2020). Proyección de poblaciones. Recuperado de https:// bit.ly/3kQdw6S 
Departamento Administrativo Nacional de Estadística (DANE). (2018). Censo Nacional de Población y Vivienda 2018 Colombia. Recuperado de https://bit.ly/3fgGv2k

Ministerio de Educación Nacional (MEN). (2019). Plan Estratégico Institucional 2019-2022. Recuperado de https://bit. ly/38ZGCya

Ministerio de Tecnologías de la Información y Comunicaciones (MinTIC). (2018a). Plan TIC 2018 - 2022 El Futuro Digital es de Todos. Recuperado de https://bit.ly/2UHUmW4

MinTIC. (2018b). Plan Nacional de Conectividad Rural. Recuperado de https://bit.ly/2UlgglT

Unesco. (5-9 de octubre de1998). World Conference on Higher Education. Higher Education in the Twenty-First Century: Vision and Action. París, Francia. Recuperado de https://bit. ly/3fjnMmO

Villatoro, S.; Silva, A. (2005). Estrategias, programas y experiencias de superación de la brecha digital y universalización del acceso a las nuevas tecnologías de información y comunicación (TIC). Un panorama regional. Santiago de Chile: Cepal. Recuperado de https://bit.ly/3pJvxY6

Wikimedia Commons (2010). Mapa del Municipio de Dabeiba, Antioquia (Colombia). Recuperado de https://bit.ly/35McHrq 